\title{
लाहौल के हिन्दू धार्मिक गीत
}

\author{
फुन्चोग डोलमा
}

पी. एच. डी. शोधार्थी, संगीत विभाग, हिमाचल प्रदेश विश्वविद्यालय, ज्ञानपथ, समरहिल, शिमला

लाहौल सामान्य परिचय

कुल्लू के उत्तर पूर्व में रोहतांग हिमद्वार के उस पार स्थित लाहौल प्रकृति का एक अद्भूत संग्रहालय है। इसमें ईश्वर ने ऐसे अद्भूत प्राकृतिक चित्रों का आयोजन किया है, जो एक ओर तो दर्शक के मन में औत्सुक्यपूर्ण भय की भावना को जगाते हैं तथा दूसरी ओर अविस्मरणीय आकर्षण उत्पन्न करते हैं। प्रकृति का यह अद्भूत सम्मिश्रण कम ही स्थानों पर दृष्टिगोचर होता है क्योंकि यहां पर एक ओर गगनचुम्बी हिमशिखर तथा सरितटों से सीधी ऊपर को उठती हुई हज़ारों फीट ऊँची काली नग्न चट्टानें हैं तो दूसरी ओर कलकल बहती हुई सरिताएं तथा उनके तटों पर बसे हुए सुन्दर मानव आवास। यहां की प्रकृति के रूप तथा मानव जीवन की विविधता प्रत्येक आगन्तुक के लिए अनन्त सम्भावनाओं के अक्षय भंडार छिपाए हुए है। भौगोलिक दृष्टि से हिमाचल प्रदेश के लाहौल-स्पिति जिले का यह उपमंडल भारत के उत्तर पश्चिम में तिब्बत सीमा के निकट पश्चिमी हिमालय की दक्षिणी ढलान पर उत्तरी अक्षांश $32^{\circ} 81^{\prime}$ तथा $32^{\circ} 59^{\prime}$ एवं पूर्वी देशान्तर $76^{\circ} 48^{\prime}$ तथा $77^{\circ} 77^{\prime}$ के बीच स्थित है। इसके उत्तर में जम्मू-कश्मीर राज्य का जिला लद्दाख, पूर्व में कुंजम हिमद्वार से विभक्त स्पिति उपमंडल, पश्चिम में ज़िला चम्बा तथा दक्षिण में ज़िला कुल्लू पड़ता है।

लाहोल का क्षेत्रफल 13835 वर्ग किलोमीटर के लगभग है, जो कि अधिकतर 18,000 फीट से भी ऊँचा पर्वतीय क्षेत्र है। चारों ओर ऊँचे-ऊँचे पर्वत शिखरों से घिरे हुए लाहुल को बाह्य जगत के साथ जोड़ने का एकमात्र दक्षिणी मार्ग रोहतांग ही है। यद्यपि इसके उतर तथा पूर्व में भी लद्दाख तथा स्पिति के साथ इसका संबंध स्थापित करने वाले बारालाचा ला तथा कुंजम हिमद्वार हैं किन्तु ये वर्ष में आधे से भी अधिक समय के लिए बंद रहता है।

लाहुल यानी ला+युल अर्थात् देवधरा, रोहतांग के उस पार आंतरिक हिमाचल क्षेत्र में बसा सुन्दर जनपद। लाहोल की यात्रा मनाली से ही आरम्भ होती है। जब आप व्यास अथवा पौराणिक विपाशा पर बने पुल से उस ओर एशिया के सर्वाधिक ऊँचे क्षेत्र से गुजरने वाली मनाली लेह राष्ट्रीय राजमार्ग को इंगित करते विशाल सूचनापट्ट को निहारते हैं तो यात्रारंभ से पूर्व ही एक अजीब सा रोमांच आपको पुलकित कर देता है। इतिहास के प्रवाह में लाहुल समय-समय पर कुल्लू एवं चम्बा के हिन्दू प्रशासकों तथा तिब्बत एवं लद्दाख के बौद्ध प्रशासकों के प्रभाव के अन्तर्गत आता रहा है। अतः यहां पर दोनों ही धर्मों का प्रभाव किसी न किसी रूप में पड़ता रहता है। किन्तु धार्मिक सम्प्रदाय के रूप में हिन्दू-धर्म के स्वरूप को मुख्यतः आर्यवंश के लोगों के द्वारा तथा बौद्धधर्म को मंगोल वंशी लोगों के द्वारा अपनाया जाता है। यद्यपि इसके अपवाद भी पाए जाते हैं। आर्यवंश के लोगों की स्थिति मुख्य रूप से चन्द्रभागा घाटी में पाए जाने के कारण इसकी परम्पराएं भी अधिकतर इसी क्षेत्र तक सीमित है। अन्य क्षेत्रों में यद्यपि उनके लोक-विश्वासों एवं कर्मकाण्ड पर हिन्दू प्रभाव परिलक्षित होता है किन्तु 
उन्हें किसी रूप में भी हिन्दू धर्म की परिधि में नहीं रखा जा सकता है। लाहुल में बौद्ध धर्म के प्रबल प्रभाव के कारण इस क्षेत्र में हिन्दू देव-मन्दिरों की संख्या बहुत ही कम है। परन्तु फिर भी चन्द्रभागा घाटी में प्राचीन काल से ही हिन्दू धर्माबलम्बियों की सत्ता तथा अनेक छोटे-बड़े मंदिर पाए जाते हैं। हिन्दू धर्मानुयायी धार्मिक गीतों, गाथागीतों के द्वारा देवी-देवताओं की स्तुति करते हैं।

धर्म और संगीत का पारस्परिक संबंध

प्राचीनकाल से ही भारतीय संगीत प्रत्यक्षतः धर्म से जुड़ा हुआ है। चूंकि सभी धर्मों का अन्तिम लक्ष्य मोक्ष प्राप्ति है और अन्य कलाओं की भांति संगीत कला का भी चरम लक्ष्य परम आनंद और पूर्ण पार्थिव अवस्था की अनुभूति कराना है। मोहमाया रूपी भवसागर से मुक्ति, आत्मा का परमात्मा से मिलन, परम शान्ति तथा अन्ततः मोक्ष प्राप्त करना ही उसका प्रमुख ध्येय माना गया है। सामवेद का संगीत भारत के इतिहास का प्रथम भक्ति-संगीत होने का अधिकारी है। यह वह संगीत है, जिसमें भक्त अपने आराध्य के प्रति अद्भुत श्रद्धा की भावनाओं को स्वरों में बांधता है। इन गीतों की सरसता का मूल उनकी आत्मीयता तथा आत्म-निवेदन की भावना है। भारत में भक्ति संगीत का प्रवाह आरम्भ से ही रहा है और कालान्तर में विकसित होने वाले धर्म भी उससे अछूते नहीं रहे। सभी प्रदेशों में समानरूपेण संगीत की व्यापकता पाई जाती है। भले ही स्थान अलग हो, परन्तु संगीत की मूलभूत भावना सभी प्रदेशों में एक सी ही है और सदैव ही जनता को एकता के सूत्र में आबद्ध करती रही है।

संगीत को साक्षात् ईश्वर का पर्याय भी माना गया है। आध्यात्मिक उद्गारों के प्रकटीकरण का माध्यम मानकर संगीत की उपासना भी की गई है। ईश भक्ति की कोई तार्किक चर्चा नहीं अपितु मनःस्थिति, आत्मिक अनुभूति एवं आस्था का विषय है एवं इस अवस्था की अनुभूति हेतु संगीत सर्वाधिक सबल माध्यम माना गया है। प्राचीनकाल में वेदों द्वारा ईश्वर प्राप्ति के तीन मार्ग निश्चित किए गए-ज्ञानमार्ग, कर्ममार्ग तथा उपासना मार्ग। इनमें से उपासना मार्ग अत्यंत सहज तथा ईश्वर से सीधा सम्पर्क स्थापित करने का सरल मार्ग है। संगीत ने उपासना मार्ग को ही अपनाया। संगीत से अपने अलौकिक प्रभावों को अनुभव किया गया, जिसे स्वानुभूति द्वारा परमानंद की अवस्था कहा गया। यह परम आनंद में डूबने का सहज माध्यम है। यह मात्र मनोरंजन नहीं है, यह सगुण से निर्गुण, अपूर्ण से पूर्ण, भौतिक से भौतिकेत्तर की ओर एक अनन्त एवम् सुमधुर यात्रा है। संगीत नादब्रह्म से एकाकार हो जाने की उर्ध्वमुखी साधना है। संगीत में डूबा संगीतकार नादब्रह्मा की उपासना, अभ्यर्थना करता है और उनके लिए राग-रागनियां, ताल, तान, स्वर, आरोह-अवरोह आदि अभिव्यक्ति मात्र है। संगीत की निर्ईरिणी तो अन्तर से बहती हुई ब्रह्म तक पहुंचती है। इसी आधार पर प्रत्येक भारतीय साधक कलाकार अपनी कलाकृति या रचना की उत्कृष्टता में केवल परमात्मा को देखता है। इसमें वह सांसारिकता से ऊपर उठकर बह्म-स्वाद-सहोदर संगीतानंद को प्राप्त करता है। यह संभजनीय गुण ही सत्य है, सत्य ही शिव है और वहीं अनंत सौन्दर्य का स्वामी है। इसलिए संगीत को बह्म विद्या कहा गया है और ईश्वर तथा उसकी महान कृति-प्रकृति से उसका सामंजस्य स्थापित किया गया है। संगीत से सद्भावना तथा साधुभाव उत्पन्न होते हैं जो कि धर्म के पावन स्तम्भ के बुनियादी घटक हैं। प्राय: सभी देशों में किसी भी धर्म के लिए संगीत द्वारा जनता के मन पर प्रभाव डालने के लिए उसे एक शक्तिशाली माध्यम के रूप में स्वीकार किया गया है। स्वर-लय में निबद्ध शब्द अत्यंत शक्तिशाली हो जाते हैं। वे 
लोगों के मन में प्रविष्ट होकर उनकी स्मृति पटल पर अंकित होकर उनकी विचारधारा को प्रभावित करते हैं। इसलिए ज्ञानोपयोगी अथवा उपदेश-संबंधी बातें गद्य की अपेक्षा प्रायः पद्य में अधिक पाई जाती है। गीता, महाभारत, रामचरितमानस, गुरूग्रन्थ साहिब आदि सभी प्रमुख धार्मिक ग्रंथ पद्य में है और इनका एक-एक शब्द दीर्घकाल से लोगों के मन को अमरत्व प्रदान करता आ रहा है।

रागबद्ध धार्मिक साहित्य के अतिरिक्त ऐसी रचनाएं भी प्राप्त हैं, जो गेय काव्य से है और केवल धार्मिक होने के नाते विशेष पूजा-अर्चना के निमित लिखी गई है। जैसे घरों में प्रचलित भजन और कीर्तन के संग्रह जो प्रसिद्ध कवियों के द्वारा नही रचे गए थें, वरन उस समय गायन में प्रचलित थे। ईश्वर की पूजा के लिए लिखी गई सूक्तियां स्तुति के लिए अनेक कविताओं और आरती गीतों की रचनाएं हुई। इसके अतिरिक्त कुछ देवी-देवताओं के गीत अपना अलग स्थान और महत्व बनाए हुए है। अतः संगीत एवं धर्म का पारस्परिक संबंध है तथा दोनों ही ऐसी समानान्तर शाखाएं हैं जो आवश्यकतानुसार एक-दूसरे को आश्रय प्रदान करती रही है। जहां धर्म के माध्यम से संगीत में सुसंस्कार, पवित्रता एवं प्रांजलता आई, वहीं संगीत के माध्यम से धर्म में सरसता आई तथा भावनाओं में मधुरता आई। संगीत को माध्यम बनाकर भगवद् आराधना करने पर पूजा-अर्चना में भाव एवं आनंद में वृद्धि हो जाती है। संगीत की इसी शक्ति को पहचान कर सम्पूर्ण विश्व में धर्मों के प्रचार-प्रसार हेतु संगीत का प्रयोग किया अर्थात् यह संगीत की संवादात्मक शक्ति से जुड़े हैं।

\section{लाहौल में हिन्दू धर्म}

लाहोल-स्पिति जनजातीय क्षेत्र का लाहोल और स्पिति वैसे तो एक ही जिला है, परन्तु इन दोनों क्षेत्रों में भी सांस्कृतिक दृष्टि से काफी भिन्नता नज़र आती है। वहीं धर्म के क्षेत्र में भी जहाँ स्पिति में केवल बौद्ध धर्म की मान्यता है, तो दूसरी तरफ लाहौल में हिन्दू एवं बौद्ध दोनों धर्मों को महत्व दिया जाता है। वर्तमान में लाहौल में मुख्यतः दो धर्मों का ही प्राधान्य है। लाहौल की पट्टन घाटी में हिन्दू धर्म का अधिक प्रचलन है, जबकि भागा और चन्द्राक्षेत्र में हिन्दू धर्म और बौद्ध धर्म का मिला-जुला रूप है। लाहोल में कई हिन्दू मन्दिर हैं, जिसमें दोनों धर्मों के लोग श्रद्धा से दर्शन करने जाते हैं। त्रिलोकीनाथ का मंदिर एवं महिषासुर मर्दिनी मृकुला देवी का मंदिर पूरे हिमाचल में प्रसिद्ध है। इसके अतिरिक्त जाहलमा का हिडिम्बा मंदिर, गोशाल का नाग मंदिर एवं शिव मंदिर प्रसिद्ध हैं। गाँव में लोगों के अपने-अपने कुल देव के मंदिर हैं, जहाँ स्थानीय लोग हर शुभ-अशुभ अवसर पर भोग चढ़ाते हैं। लाहौल में भी हिन्दू मन्दिरों में भजन-कीर्तन का समय तय होता है। लोग अपनी इच्छापूर्ति के लिए अपने कुल देवी-देवता के नाम पर जागरण कर पूरी रात उनका आह्वान करते हैं। गाँव जाहलमा निवासी श्री शोभाराम जी के अनुसार घरों में जब जगराता का अनुष्ठान होता है तो सुबह प्रोहातराग 2:00 से 2:30 बजे तक फिर बट्टबाण राग, इसके पश्चात् रात को निन्द्राली राग बजाई जाती है। त्रिलोकनाथ मंदिर की एक विशेषता यह भी है कि इस मंदिर का पुजारी बौद्ध लामा हैं, परन्तु मंदिर का मुखिया ठाकुर एक राजपूत है। मंदिर में सूर्योदय और सूर्यास्त के समय पूजा और आरती हिन्दू पद्धति के अनुसार ढोल, नगारा, रणसिंगा आदि वाद्ययंत्रों के साथ होती है। 
लाहौल के हिन्दू धार्मिक गीत

लाहौल-स्पिति के लोगों का सामाजिक जीवन, धार्मिक भावना से ओत-प्रोत है। यहां के लोगों में धार्मिक सहिष्णुता, धर्म के प्रति श्रद्धा, विश्वास एवं भक्ति की भावना कूट-कूट कर भरी है। यहां के लोग अनेक देवी-देवताओं की पूजा करते हैं, जिनमें शिव-पार्वती, श्रीराम, श्री कृष्ण आदि प्रमुख हैं। इन देवी-देवताओं से सम्बन्धित यहां कई प्रकार के गीत प्रचलित है-

क) पाण्डवों से सम्बन्धित गीत

जब थीए राजा पाण्डुए

तब थीए नौ गज माहणू हो

जब थीए राजा पाण्डुए, तब थीए सतगज जनाना हो

जब थीए राजा पाण्डुए, तब थीए घोडाये सींगा हो

जब थीए राजा पाण्डुए, तब थीए काकुल जोआन हो

जब थीए राजा पाण्डुए, तब थीए माहणू जोआना हो।

भावार्थ- प्रस्तुत गाथागीत का भावार्थ है कि जब धरती पर पाण्डवों का राज्य था तब मानव नौ गज लम्बे थे, उस समय स्त्रियां सात गज लम्बी थी। आज की अपेक्षा उस काल में पशु-पक्षी और मनुष्य भिन्न थे। उस समय घोड़े के सींग होते थे। अनाज का एक दाना एक सेर के बराबर का था। इसी प्रकार अन्य सभी चीज़ें भी आज से भिन्न थी।

ख) शिव से सम्बन्धित गीत

शिव से सम्बन्धित इस गीत में शिव तथा पार्वती एवं गणेश जी की महिमा का वर्णन किया गया है-

शिवा गोरी ए बड़ी झगुडा कीति

गोरी राणी ए रूशी कारी गेयी

रूशिकारी गेयी समुन्दरा कान्टे

शिवा गोरी ए बड़ी झगुडा कीति

गोरी राणी ए बेसुता होई

गोरी राणी ए माणा विषा खाए

जट्टाधारी ए खबुरा होई

शिवा जट्टाधारी समुन्दरा कान्टे

शिवा बोलनदा क्या धौरा कराणा

ब्रह्म वीरा शादी कारी आणी

ब्रह्म वीरा गरूणा श्धाये

गरूणा राजा सेने पंखा फेरी

सोना पंखा विषा आड़ी

गोरी राणीए होशा आयी। 
भावार्थ- प्रस्तुत गीत में शिव और पार्वती के झगड़े का उल्लेख किया गया है। एक बार पार्वती अपने कमरे में स्नान कर रही थी तो उसी समय शिव भी वहां आ गए और अन्दर जाने लगे परन्तु गणेश ने उन्हें अन्दर आने से मना कर दिया। जिससे भगवान शिव ने क्रोधित होकर गणेश का सिर काट डाला। पार्वती अपने पुत्र का वध देखकर बहुत दुखी हुई और उन्होंने आत्महत्या हेतु एक मण जहर खा लिया। यह सब देखकर ब्रह्मा जी बहुत अधीर हुए और उन्होंने गरूड़ की सहायता से विष को खत्म कर दिया। उस समय भगवान शिव ने यह वचन दिया कि किसी भी अनुष्ठान में गणेश जी का स्थान प्रथम रहेगा।

ग) अनेक देवी-देवताओं से सम्बन्धित गीत

ब्रह्म वीरा मेसरा नावें

मेसरा नावें सरस्वती नारी

सरस्वती नारी ब्रह्म ओ प्यारी

शिव शंकरा पार्वती नारी

पार्वती नारी शिवशंकरा प्यारी

पांडवा राजे धर्मों राजे

मनुषा जूने गौ पैदा नाहि

माता कुन्ती डाणून्दो लागो

जिन्दे मलाणी ओतारी नहीं

मुर्दे मलाणी पवित्रा नहीं

जनों भीइयां द्वारिकापुरी

भोई ओ वीरा दौडुन्दे लागी

अधु बाते आया द्वारिकापुरी

श्रीकृष्ण आया भीईयां ढूंणा चूणा किति

भीईयां ओ वीरा अरूजन कीति

माता और कुन्ती डाणुन्दे न लागी

श्रीकृष्ण त भीइयां विन्दुरा वाणे।

भावार्थ- प्रस्तुत गीत में ब्रह्म, महेश्वर, श्रीकृष्ण, शिव तथा पाण्डवों का उल्लेख देखने को मिलता है। यह गीत शिव और पार्वती, पाण्डवों और माता कुन्ती, ब्रह्मा और सरस्वती के संबंधों को लेकर अच्छी-खासी चर्चा करता है। गीत में माता कुन्ती की मृत्यु की दशा बताई गई है तथा माता कुन्ती को यह भी खबर है कि बिना गऊ माता दर्शन के उनको मुक्ति नहीं मिल सकती इसलिए अपनी मुक्ति के लिए वह भीम को द्वारिका से गाय लाने के लिए भेजती है। भगवान कृष्ण भीम के इस संदेश से वृन्दावन में गाय की तलाश में जाते हैं। सर्वप्रथम उन्हें गाय के खुर के चिन्ह दिखाई देते हैं। इनके दिखने के बाद वह इस पर अपना माथा टेकते हैं। थोड़ी दूरी पर गोबर का ढेर भी दिखाई पड़ता है। आगे चलते-चलते कुछ दूरी पर उन्हें गौ माता के दर्शन हो जाते हैं। वह गौ माता से हस्तिनापुर चलने की प्रार्थना करते हैं। पहले तो वह इनकार करती हैं लेकिन आखिर में चलने का वचन देती है। अंत में बहुत ही कठिन परिश्रम के बाद उन्हें गाय माता मिल जाती हैं तो भीम गाय 
को पीठ में उठाकर हस्तिनापुर पहुंच जाता है। माता कुन्ती गौ माता के दर्शन करती है और मुक्ति को प्राप्त करती है। इस तरह से माता कुन्ती का अन्तिम संस्कार सम्पन्न किया जाता है।

घ) गुरू घटांल मठ से सम्बन्धित गीत

उपरू घण्टाले री डोशा भुई

तान्दी घुशाले साला विगुड़ी

उपरू घण्टाले लामा गुरू नाही

त्रिज्जी ओ लम्वूरा शदि कारी आणी

त्रिज्जी ओ लम्वूरा केलांगा भेजी

त्रिज्जी ओ लम्वूरा केलांगा कोठी

ठाकुरू हिरा चन्दा केलांगा कोठी

ठाकुरू हिरा चन्दा पुछुंणे जो दीति

त्रिज्जी ओ लम्वूरा जुवाबा जो दीति

उपरी घंटाले री डोशा भुई

तादी घुशाले साला विगुडी

उपरू घण्टाले लामा गुरू नाही

ठाकुरू हिरा चन्दा हुकुमा जो दीति

त्रिज्जी ओ लम्वूरा हुकुमा जो सुणदे

जोडी ओ जोडी लादाखा जो पहुचे

टशी थम्पेला पूछणे लागी

जोडी ओ जोडी जुवावा जो दीति

असे दूए तऊ जो शदूणे जो आए जी।

भावार्थ- प्रस्तुत लोकगाथा गीत में गुरू घण्टाल गोम्पा के प्रमुख लामा का गोम्पा छोड़कर जाने की कथा है। गोम्पा में पूजा-अर्चना की अनिश्चितता के कारण मुख्य लामा, लोगो से नाराज़ होकर लद्दाख चले जाते है। लामा के चले जाने से गौशाला और तांदी गांव की फसलों का बहुत ही बुरा हाल होता है। यहां के लोगों की भूखे मरने वाली स्थिति पैदा हो जाती है इतनी दुर्दशा होने से अंत में विवशता यहां के तीन पंचायत प्रधानों की बैठक होती है जिसमें यह तय किया जाता है कि मुख्य लामा को वापिस बुलाया जाए। जब तक उन्हें नहीं बुलाया जाता तब तक हमारी यह दशा ज्यों की त्यों ही बनी रहेगी। इसलिए उनको बुलाने के लिए दो व्यक्तियों को नियुक्त किया जाता है। जब लामा जी वापिस लाहोल पहुंच जाते हैं तो यहां की स्थिति फिर से सामान्य हो जाती है।

घ) प्रभु गोरखनाथ जी से सम्बन्धित गाथा गीत

अन्चेला बन्चेला दुये जोड़ी बेणी जी

भोला बन्चेला गुरू दाने ब्याही जी

भोला बन्चेला पुतुरा नाही जी

बारह हो बरसे जवे तापे बैठी जी 
गुरू ओ गोरखनाथ बन्चूले री प्रोढी जी

कोढी भेषे गोरखनाथ बन्चूले री दवारे जी

भोला बन्चेला गुरू सेवा पूजा कीति जी

पुछुदे गुरू गोरखनाथ क्या बचना तऊ लोड़ी जी

मंगदे भोल बन्चेला पुतुरा फला जी

माघा ओ महीने जेठी ओ अईतवारा जी

गोरखू टिला जी दर्शना अऊँणा जी

माघा ओ महीने जेठी ओ अईतवारा जी

भोला बन्चेला गोरख टीले जो तैयारी जी

सोने री थाड़ी जो भोग न कीति जी

दूजी ओ गुगला रे घूपा जी

भोला ओ बन्चेला गोरखू टिला बैठी जी

बारह बरसे जपे-तपे कीति जी

भोला ओ बन्चेला रोलणे जो लागी

एकीओ टीपू आखरो गोरखनाथे री पीठे

कागओ पण्डिता गोरूखा टिला भेजी जी

खबुरा सुणादे कागा ओ पण्डिता जी

देखा ओ गुरू मेरे सितुरी जापे तापे जी

एकी हाथे सोने थाड़ी दूजी हाथे गुगुला धुपा जी

गुरू गोरखनाथे दर्शना दीति जी

खुशी-खुशी बन्चेला फला पुतुरा दीति जी

भोला बन्चेला मस्तुका टेकी जी

तेटी कना भोला बन्चेला घरे जोगू आए जी।।

भावार्थ- प्रस्तुत गाथा में बौद्ध धर्म से सम्बन्धित एक बांझ स्त्री को प्रभु गोरखनाथ की कृपा से पुत्र फल की प्राप्ति की कथा का वर्णन मिलता है। यह लोक गाथा यह बताती है कि मनुष्य अगर अपने काम को निःस्वार्थ भाव से करेंगे तो वह अवश्य ही एक दिन अपनी इच्छा को प्राप्त करते हैं। इस गीत के माध्यम से यह भी बताया गया है कि हमें अपने दुःख से अधिक महत्व दूसरों के दुःख को देना चाहिए। प्रस्तुत गाथा में स्त्री बन्चेला स्वयं अत्यधिक दुखी होते हुए भी अपने दरबार पर आए कोढ़ी व्यक्ति की दिलोजान से सेवा करती है। यह कोढ़ से पीड़ित व्यक्ति साधारण व्यक्ति नहीं होता अपितु, भगवान गोरखनाथ स्वयं इस रूप में उसकी परीक्षा लेने आए होते हैं। अंत में भगवान इस परिणाम पर पहुंचते हैं कि वह स्त्री अपनी परीक्षा में सफल है, और प्रभु उससे प्रसन्न होते हैं। इसमें बन्चेला नाम की स्त्री की लगन और मेहनत एवं आस्था देखकर एकदिन प्रभु गोरखनाथ उन्हें प्रत्यक्ष रूप में दर्शन देते हैं और उससे वर मांगने को कहते हैं। स्त्री उनसे कहती है, हे प्रभु तुम्हें तो सबकुछ मालूम है। मुझे तुम्हारे ही नाम का आसरा है। प्रभु उसके मन की बात जान लेते हैं और उसे पुत्र फल देते हैं। 


\section{उपसंहार}

संगीत ईश्वर की वह शक्ति है, जो प्रकृति के कण-कण में समाहित है। सृष्टि के स्वर्णिम ब्राह्मण से प्रलय की काली संध्या तक संगीत का अस्तित्व स्वीकार्य है। अतः संगीत का क्षेत्र व्यापक है, अनादि है तथा अनन्त है। हिमाचल प्रदेश की सांस्कृतिक विरासत यहां के लोकसंगीत के माध्यम से मुखरित हुई है। अतः यहाँ की प्राचीन सभ्यता एवं सांस्कृतिक धरोहर को प्रमाणित करने की दिशा में लोकगीतों, लोकनतत्यों एवं लोकवाद्यों का अत्यंत महत्त्वपूर्ण स्थान है। हिमाचल के आंचल में बसा जनजातीय ज़िला लाहोल-स्पिति अपनी सांस्कृतिक धरोहर को पीढ़ी-दर-पीढ़ी संजोए विकास के पथ पर अग्रसर है। इसमें धर्म का महत्त्वपूर्ण स्थान है। लाहौल-स्पिति का लाहौल क्षेत्र अपने दोनों धर्मों की मान्यता के लिए प्रसिद्ध है। इसका साक्षात् उदाहरण त्रिलोकनाथ का मंदिर है जहाँ हिन्दू धर्म के श्रद्धालुओं की भी उतनी ही भीड़ होती है जितनी कि बौद्ध धर्म के श्रद्धालुओं की।

लाहौली समाज में सबसे पहले 7 वीं शताब्दी में हर्षवर्धन के काल में हिन्दू और बौद्ध धर्म का मिला-जुला रूप देखा गया। लाहौल के पट्टन घाटी में हिन्दू धर्म पूर्ण रूप से प्रचलित है। यहाँ के लोग शिव, नाग को अधिक पूजते हैं। यहाँ पर आज भी हिन्दू धर्म से सम्बन्धित कई दंत कथाएं प्रचलित हैं। गाँव के बुजुर्ग दादरा, खेमटा आदि ताल में कृष्ण, राम, शिव-पार्वती, ब्रह्म आदि के गीत गाते हैं। घरों में जागरण के दौरान भी इन गीतों का गायन होता है। मंदिरों में पूरे हिन्दू रीति-रिवाज़ से भजन कीर्तन होते हैं। वास्तव में यह सारा जनजातीय क्षेत्र सहनशीलता, सहिष्णुता, सर्वधर्म-समानता, सहानुभूति का उत्कृष्ट संगम स्थल है। यहाँ की जनता सद्भावना, आपसी भाईचारा, करूणा और संयम की प्रतिमूर्ति है। हिन्दू धर्म की मर्यादा दिन प्रतिदिन फलती-फूलती जा रही है जिसे हमारे बुजुर्गों ने नई पीढ़ी के लिए संरक्षित रखा है।

\section{सन्दर्भ ग्रन्थ सूची}

कुमार अशोक, संगीत और संवाद, कनिष्क पब्लिशर्स नई दिल्ली, 2007

ठाकुर सूरत, हिमाचल के लोकवाद्य, अभिषेक पब्लिकेशन्ज चण्डीगढ़, 1996

विशिष्ट सुदर्शन, लाहौल-स्पिति, हिमाचल कला संस्कृति भाषा अकादमी शिमला, 2003

शर्मा डी.डी., हिमालय की विर्मय भूमि लाहुल, इरा प्रकाशन पंत कुटीर सोलन, 1998

शर्मा भारती, सांगीतिक एवं धार्मिक परम्परा, संजय प्रकाशन नई दिल्ली, 2006

शर्मा मनोरमा, हिमाचल (संस्कृति एवं परम्परा), आउट लाइन पब्लिशर्ज दमशेश नगर दिल्ली, 2012 सचदेव रेनू, धार्मिक परम्पराएं एवं हिन्दुस्तानी संगीत, राधा पब्लिकेशन्स नई दिल्ली, 1999

सिन्हा ज्योति, संगीत सारांश, ओमेगा पब्लिकेशन्स नई दिल्ली, 2012 